\title{
15 Jahre Transfusionsgesetz - eine Erfolgsgeschichte
}

Das deutsche Transfusionsgesetz (TFG) von 1998 [1] geht auf die weltweite Medizinkatastrophe der Übertragung des Humanen Immundefizienz-Virus (HIV) durch Blutprodukte zurück. Allein in Deutschland wurden mehrere hundert Menschen in Mitleidenschaft gezogen. Der Untersuchungsausschuss des Deutschen Bundestages „HIV-Infektionen durch Blut und Blutprodukte" hat in seinem Abschlussbericht von Oktober 1994 [2] ein in sich geschlossenes Transfusionsgesetz gefordert. Dieser Forderung ist der Gesetzgeber mit dem Transfusionsgesetz von 1998 gefolgt, das zu der Zeit einzigartig in der Europäischen Gemeinschaft war. Es war Vorbild für die später folgenden verbindlichen Richtlinien der Europäischen Gemeinschaft zu den Qualitäts- und Sicherheitsstandards für Blut und Blutbestandteile aus den Jahren 2002, 2004 und 2005 [3-6].

\section{Das Transfusionsgesetz als Ergänzung zu Arzneimittelgesetz und Arzneimittelrecht $\nabla$}

Das TFG ist neben die Vorschriften des Arzneimittelgesetzes (AMG) [7] und des übrigen Arzneimittelrechts, insbesondere der Arzneimittel- und Wirkstoffherstellungsverordnung (AMWHV) [8], getreten, die schon vorher für Blutzubereitungen im Sinne von $\S 4$ Abs. 2 AMG galten. Ziel ist nach $\S 1$ TFG die sichere Gewinnung von Blut und Blutbestandteilen zur gesicherten und sicheren Versorgung der Bevölkerung mit Blutprodukten. Insbesondere die Anforderungen des 2. Abschnitts (Gewinnung von Blut und Blutbestandteilen) und des 4. Abschnitts (Rückverfolgung) des TFG ergänzen die Vorschriften des Arzneimittelrechts und müssen erfüllt werden, um die arzneimittelrechtliche Herstellungserlaubnis zu erlangen ( 0 Kasten 1). Zusätzlich kann die Bundesärztekammer (BÄK) zusammen mit der zuständigen Bundesoberbehörde den allgemein anerkannten Stand der medizinischen Wissenschaft und Technik nach $\S$ 12a TFG feststellen [9]. Zuständige Bundesoberbehörde ist nach $\S 27$ Abs. 1 TFG das Paul-Ehrlich-Institut (PEI). Der Arbeitskreis Blut nach § 24 TFG erarbeitet Voten und Stellungnahmen zu aktuellen Themen und Fragestellungen.

Kasten 1:
Abschnitte des TFG
- Erster Abschnitt: Zweck des Gesetzes,
Begriffsbestimmungen
- Zweiter Abschnitt: Gewinnung von
Blut und Blutbestandteilen
- Dritter Abschnitt: Anwendung von
Blutprodukten
- Vierter Abschnitt: Rückverfolgung
- Fünfter Abschnitt: Meldewesen
- Sechster Abschnitt: Sachverständige
- Siebter Abschnitt: Pflichten der
Behörden
- Achter Abschnitt: Sondervorschriften
- Neunter Abschnitt: Bestimmung der
zuständigen Bundesoberbehörden
und sonstige Bestimmungen
- Zehnter Abschnitt: Straf- und Buß-
geldvorschriften
- Elfter Abschnitt: Übergangsvor-
schriften
- Zwölfter Abschnitt: Schlussvor-
schriften

Die Ermächtigung in Artikel 74 Abs. 1 Nr. 26 GG hat es dem Gesetzgeber erlaubt, in das Transfusionsgesetz auch Vorschriften zur Anwendung von Blutprodukten aufzunehmen (3. Abschnitt). Auch mit diesen Vorschriften wird das Ziel der sicheren, insbesondere qualitätsgesicherten Versorgung der Patienten mit Blutprodukten verfolgt. Dazu dienen klare Struk- turen und grundsätzliche Vorgaben zur Behandlung. Die Konkretisierung der rechtlichen Grundsätze zur Anwendung der Blutprodukte ist vor allem der Bundesärztekammer nach § 18 TFG aufgegeben und erfolgt in Richtlinien als allgemein anerkannter Stand der medizinischen Wissenschaft und Technik und in Therapieleitlinien [10].

Schließlich verfolgt das TFG das Ziel der gesicherten Versorgung mit Blutprodukten und hat dabei auch den Aspekt der europäischen Selbstversorgung im Blick, um in Fällen der Verbreitung von gefährlichen Krankheitserregern in anderen Teilen der Welt autark in der Versorgung sein zu können.

Im Folgenden soll anhand der genannten Bereiche

- Sicherheit der Blutprodukte und

Hämovigilanz

- sichere Anwendung von

Blutprodukten und

- gesicherte Versorgung mit

Blutprodukten

deutlich gemacht werden, wie sich das TFG ausgewirkt hat.

\section{Sichere Versorgung durch sichere Blutprodukte \\ $\nabla$}

Es besteht kein Zweifel, dass heute in Deutschland aufgrund der europäischen und innerstaatlichen Vorschriften sowie des hohen allgemein anerkannten Standes der medizinischen Wissenschaft und Technik die verfügbaren und zur Anwendung kommenden Blutprodukte höchsten Qualitäts- und Sicherheitsanforderungen genügen. Wie $\odot$ Tab. 1 des RKI zeigt, ist die Infektionshäufigkeit bei Neuspendern und Mehrfachspendern heute äußerst gering. Die Zahlen bewegen sich auf einem konstant niedrigen Niveau.

Experten schätzen das Restrisiko, durch eine Blutspende mit HIV infiziert zu werden, auf eine Infektion zu 9,64 Mio. Transfusionen (1:9,64 Mio.) bei Durchführung einer Antikörpertestung und der NATPool-Testung (NAT: Nukleinsäure-Amplifikations-Techniken) ein. Bei Durchführung der Antikörpertestung und der NATEinzeltestung ist das Restrisiko für eine HIV-Infektion sogar nur 1:19,28 Mio. Damit ist die Wahrscheinlichkeit, durch Blutprodukte mit HIV infiziert zu werden, heute verschwindend gering. Im Vergleich dazu wurde 1997 das Restrisiko 
Tab. 1 Infektionsepidemiologische Eckdaten von Blutspendern 2008-2010.

\begin{tabular}{|c|c|c|c|c|c|c|c|c|c|c|c|c|}
\hline & \multicolumn{4}{|c|}{2008} & \multicolumn{4}{|c|}{2009} & \multicolumn{4}{|c|}{2010} \\
\hline & HIV & $\mathrm{HCV}$ & HBV & Syphilis & HIV & $\mathrm{HCV}$ & HBV & Syphyilis & HIV & $\mathrm{HCV}$ & HBV & Syphilis \\
\hline Neuspender & \multicolumn{4}{|c|}{$n=570852$} & \multicolumn{4}{|c|}{$n=614109$} & \multicolumn{4}{|c|}{$\mathrm{n}=560047$} \\
\hline Infektionen & 39 & 407 & 780 & 177 & 43 & 501 & 807 & 202 & 37 & 386 & 651 & 236 \\
\hline $\begin{array}{l}\text { Prävalenz / } 100000 \\
\text { Spenden }\end{array}$ & 6,8 & 71,3 & 136,6 & 31,0 & 7,0 & 81,6 & 131,4 & 32,9 & 6,6 & 68,9 & 116,2 & 42,1 \\
\hline $\begin{array}{l}1 \text { Infektion pro } X \\
\text { Spenden }\end{array}$ & 14637 & 1403 & 732 & 3225 & 14282 & 1226 & 761 & 3040 & 15136 & 1451 & 860 & 2373 \\
\hline Mehrfachspenden/r & \multicolumn{4}{|c|}{$n=6658627 / 2377166$} & \multicolumn{4}{|c|}{$n=6945746 / 2502534$} & \multicolumn{4}{|c|}{$n=6955495 / 2514149$} \\
\hline Infektionen & 61 & 53 & 34 & 105 & 54 & 66 & 18 & 97 & 64 & 61 & 25 & 98 \\
\hline $\begin{array}{l}\text { Serokonversionen / } \\
100000 \text { Spenden }\end{array}$ & 0,92 & 0,80 & 0,51 & 1,58 & 0,78 & 0,95 & 0,26 & 1,40 & 0,92 & 0,88 & 0,36 & 1,41 \\
\hline $\begin{array}{l}1 \text { Infektion pro } X \\
\text { Spenden }\end{array}$ & 109158 & 125634 & 195842 & 63415 & 128625 & 105239 & 385875 & 71606 & 108680 & 114025 & 278220 & 70974 \\
\hline $\begin{array}{l}\text { Serokonversionen / } \\
100000 \text { Spender }\end{array}$ & 2,57 & 2,23 & 1,43 & 4,42 & 2,16 & 2,64 & 0,72 & 3,88 & 2,55 & 2,43 & 0,99 & 3,90 \\
\hline
\end{tabular}

noch auf 1:1 Mio. geschätzt. Günstige Prognosen können heute auch für andere Krankheitserreger wie Hepatitis-C-Virus und Hepatitis-B-Virus gegeben werden.

Ein wesentlicher Wert des TFG besteht darin, dass es neben den fachlichen Anforderungen die Strukturen der damals beste- henden fachlich/wissenschaftlichen Begleitung und Weiterentwicklung des Blutspende- und Transfusionswesens gesetzlich festgeschrieben hat. Das hat zu einer dauerhaften praktischen und wissenschaftlichen Aufmerksamkeit der Fachkreise geführt, die für dieses wichtige Spezialgebiet der Medizin unerlässlich ist.

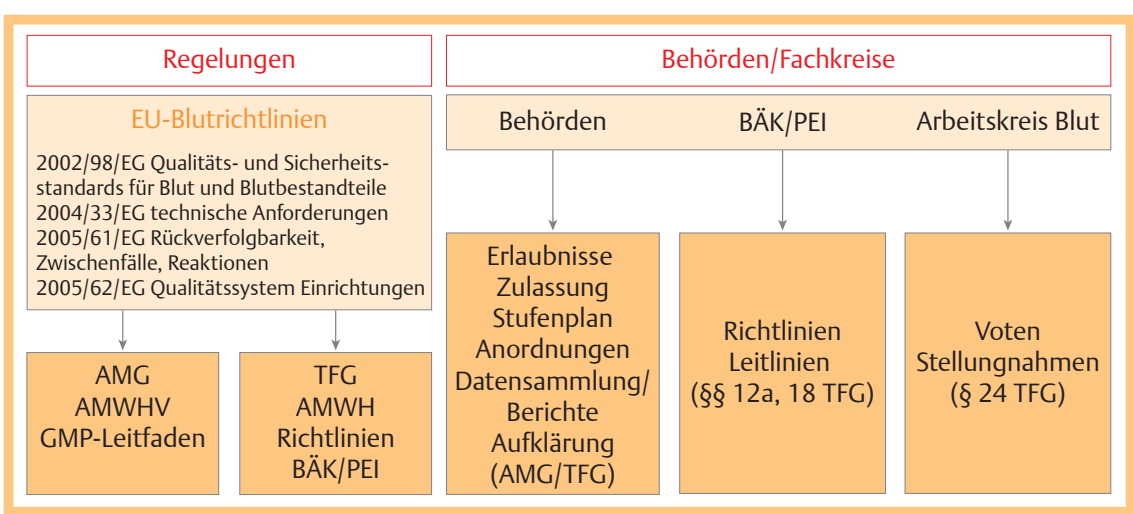

Abb.1 Regelungen und fachliche Strukturen für das Blutspende- und Transfusionswesen.

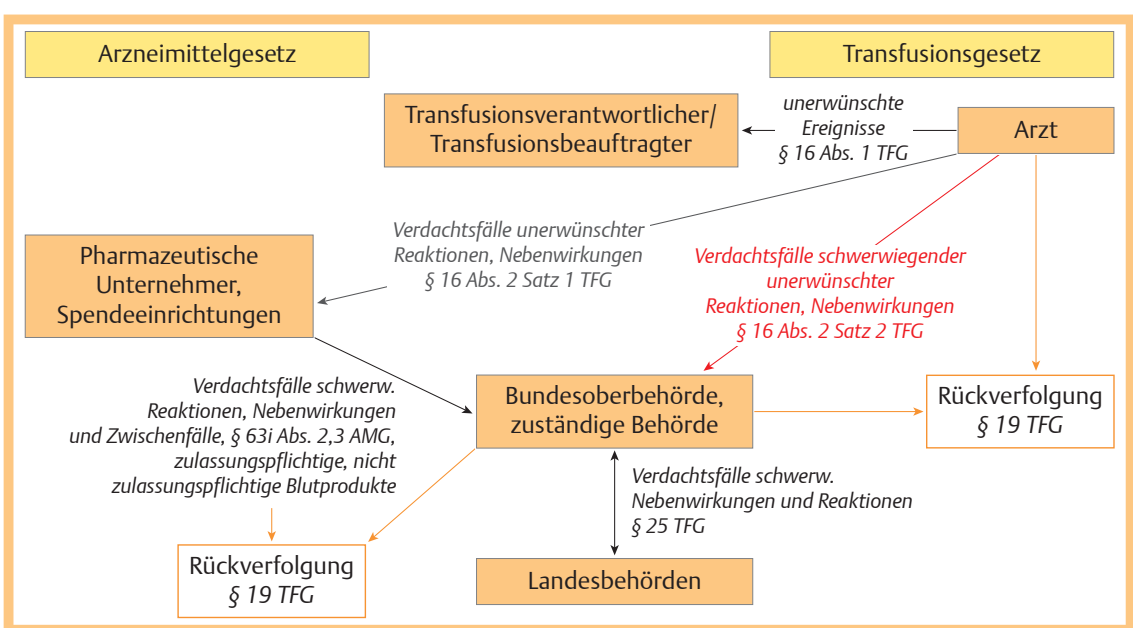

Abb. 2 Melde- und Unterrichtungswege (Quelle: PEI/von Auer).
Das gilt auch für die Arbeit der Behörden (Bundesoberbehörden - PEI, Robert KochInstitut (RKI) und die Bundeszentrale für gesundheitliche Aufklärung (BZgA), zuständige Behörden der Länder), die einen erheblichen Anteil daran haben, dass verbindliche Standards bei der Herstellung, Versorgung, Überwachung und dem Inverkehrbringen/Vertrieb von Blutprodukten eingehalten werden. $\bullet$ Abb. 1 zeigt eine Übersicht über die bestehenden Regelungen und die fachlichen Strukturen zur Festlegung und Feststellung der konkretisierenden Standards.

Es hat sich gezeigt, dass die Zusammenarbeit und die Abstimmung zwischen den Fachkreisen untereinander und zwischen den Fachkreisen und den Behörden gut funktioniert. Erkenntnisse des Arbeitskreises Blut beim RKI, der häufig Entscheidungen zu aktuellen Themen und Fragen zu treffen hat, gehen nicht selten in die Überlegungen von BÄK und PEI im Rahmen der Richtliniengebung ein. Auf das Votum 34 zur Rückverfolgung nehmen die Hämotherapierichtlinien ausdrücklich Bezug.

\section{Hämovigilanz \\ $\nabla$}

Das Konzept der Hämovigilanz hat sich bewährt. Es setzt sich aus Vorschriften des TFG und des AMG zusammen, deren Anforderungen eng miteinander verknüpft sind. Während § 63i AMG die Dokumentations- und Meldepflichten der Blut- und Plasmaspendeeinrichtungen im Falle des Verdachts schwerwiegender Zwischenfälle und Ereignisse bei zulassungspflichtigen und nicht zulassungspflichtigen Blutzubereitungen vorschreibt, legt $§ 16$ TFG die Unterrichtungspflichten der ärzt- 
lichen Personen in den Einrichtungen der Krankenversorgung für diese Verdachtsfälle fest. $\triangle$ Abb. 2 zeigt eine schematische Übersicht der Melde- und Unterrichtungswege.

§ 25 TFG verpflichtet die zuständigen Behörden von Bund und Ländern, sich bei Verdachtsfällen schwerwiegender unerwünschter Reaktionen oder Nebenwirkungen von Blutprodukten unverzüglich gegenseitig zu unterrichten. Zusammen mit den Anforderungen zur Rückverfolgung nach $\S 19$ TFG ist das Netz der Hämovigilanz dicht geknüpft. Die Erhebung der epidemiologischen Daten der Spenderkollektive nach $\S 22$ TFG dient dazu, das Infektionsgeschehen in diesen Kollektiven sorgfältig im Blick zu behalten (vgl. - Tab. 1) und mit geeigneten epidemiologischen Maßnahmen möglichen Gefahren rechtzeitig zu begegnen. Auch helfen die Ergebnisse den Spendeeinrichtungen, den Einrichtungen der Krankenversorgung, den zuständigen Landesbehörden und dem PEI bei ihren Arbeiten und Überlegungen für ein sicheres Blutspende- und Transfusionswesen. Die jährlichen Übersichten sind auf der Homepage des RKI einzusehen.

\section{Sichere Versorgung durch sichere Anwendung von Blutprodukten}

Eine Besonderheit ist der 3. Abschnitt des TFG. Darin sind die wichtigsten Grundsätze und Strukturen der Anwendung von Blutprodukten geregelt. @ Abb. 3 zeigt die Organisationsstruktur, die das Korsett der Qualitätssicherung der Anwendung von Blutprodukten in den Einrichtungen der Krankenversorgung bildet (vgl. § 15 TFG und die Hämotherapierichtlinien).

Ausführlich nehmen die Hämotherapierichtlinien der BÄK und des PEI gemäß $\S 18$ TFG zur Qualitätssicherung der Anwendung von Blutprodukten Stellung und stellen auch die Qualifikationen der verschiedenen Funktionsträger fest. Die zuständigen Ärztekammern in den Ländern haben Umsetzungsbeschlüsse zu den Hämotherapierichtlinien gefasst und Merkblätter zur Qualitätssicherung sowie Musterarbeitsanweisungen herausgegeben [11]. Zusätzlich hat die BÄK in Querschnitts-leitlinien zur Therapie mit Blutkomponenten und Plasmaderivaten Empfehlungen für die Anwendung der verschiedenen Blutprodukte und die Indikationen erarbeitet [12]. Fachverbände und

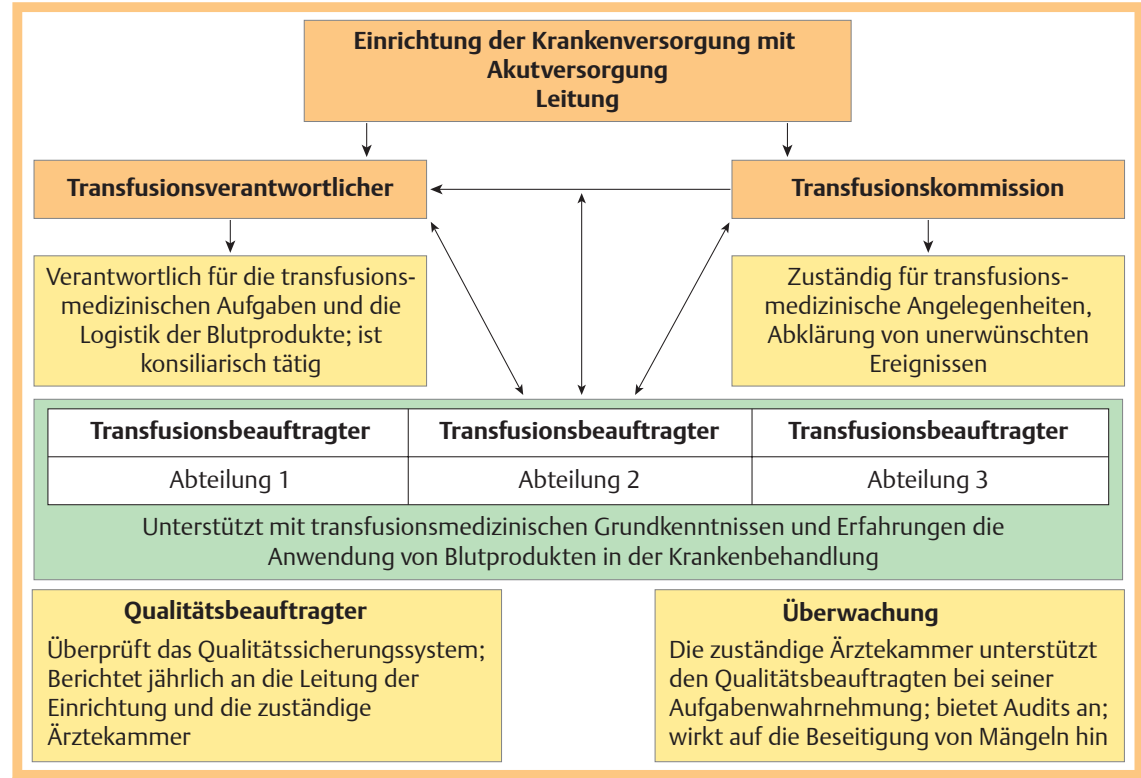

Abb. 3 Organisationsstruktur zur Qualitätssicherung bei der Anwendung von Blutprodukten in medizinischen Einrichtungen.

Universitätskliniken haben Qualitätshandbücher und Qualitätsmanagementhandbücher verfasst [13], und die Fachgesellschaften stellen immer wieder ergänzende Empfehlungen zur Verfügung. Durch Fortbildung werden junge Ärztinnen und Ärzte auf die Aufgaben als Transfusionsbeauftragte vorbereitet. Insgesamt kann von einer engagierten und verantwortungsvollen Erfüllung der Anforderungen in den Vorschriften und den ausgearbeiteten fachlichen Standards gesprochen werden, so dass ein hohes Maß an Qualität und Sicherheit bei der Anwendung von Blutprodukten in den Einrichtungen der Krankenversorgung gewährleistet ist.

\section{Gesicherte Versorgung der Bevölkerung mit Blutprodukten (Selbstversorgung)}

Nach $\S 3$ Abs. 1 TFG haben die Spendeeinrichtungen die Aufgabe, Blut und Blutbestandteile zur Versorgung der Bevölkerung mit Blutprodukten zu gewinnen. Damit hat der Gesetzgeber auch den Aspekt der gesicherten Versorgung mit Blutprodukten in den Blick genommen. Die Versorgung soll auch bei Engpässen und in Not- und Krisenfällen sicher gestellt werden. Deshalb verpflichtet der Gesetzgeber in $\S 3$ Abs. 2 TFG die Spendeeinrichtungen und ihre Träger zur Zusammenarbeit und zur gegenseitigen Unterstützung bei Versorgungsengpässen und verlangt, die Einzelheiten in einer Vereinbarung festzule- gen. $\triangle$ Kasten 2 gibt die Inhaltsangabe der Vereinbarung der Träger der Spendeeinrichtungen vom 29.05.2008 wieder (siehe unter www.stkb.de/Verband/Publikationen). Sie dient auch der Gewährleistung eines fairen Wettbewerbs im Blutspendewesen.

Kasten 2:

Rahmen für eine Vereinbarung nach $\S 3$ Abs. 2 Satz 3 des

Transfusionsgesetzes zwischen den Spendeeinrichtungen in der Bundesrepublik Deutschland

\section{Präambel}

- Artikel I. Versorgung von Einrichtungen der Krankenversorgung ohne eigene Spendeeinrichtungen

- Artikel II. Zusammenarbeit der Spendeeinrichtungen

- Artikel III. Werbung und Blutspendeaktionen

Artikel IV. Blutspendedienst der Bundeswehr

Artikel V. Beratungskommission Blutversorgung

- Artikel VI. Schlussbestimmungen

Zur besseren Einschätzung des Versorgungsgrades und für mögliche Maßnahmen der Behörden werden Zahlen zur Gewinnung von Blut und Plasma und zur Herstellung von Blutprodukten sowie zur Anzahl der Patienten mit Hämostasestörungen gesammelt, zusammengestellt und einmal jährlich veröffentlicht. Die Spendeeinrichtungen übermitteln dafür 


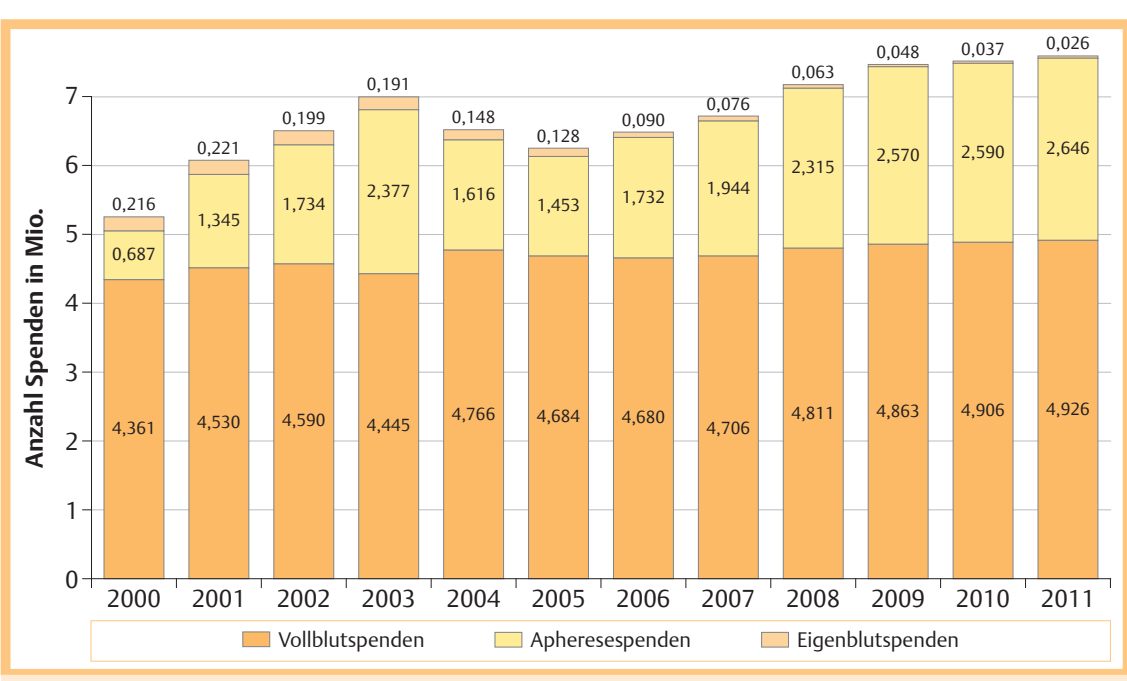

Abb. 4 Entwicklung des Spendenaufkommens (Quelle: PEI)

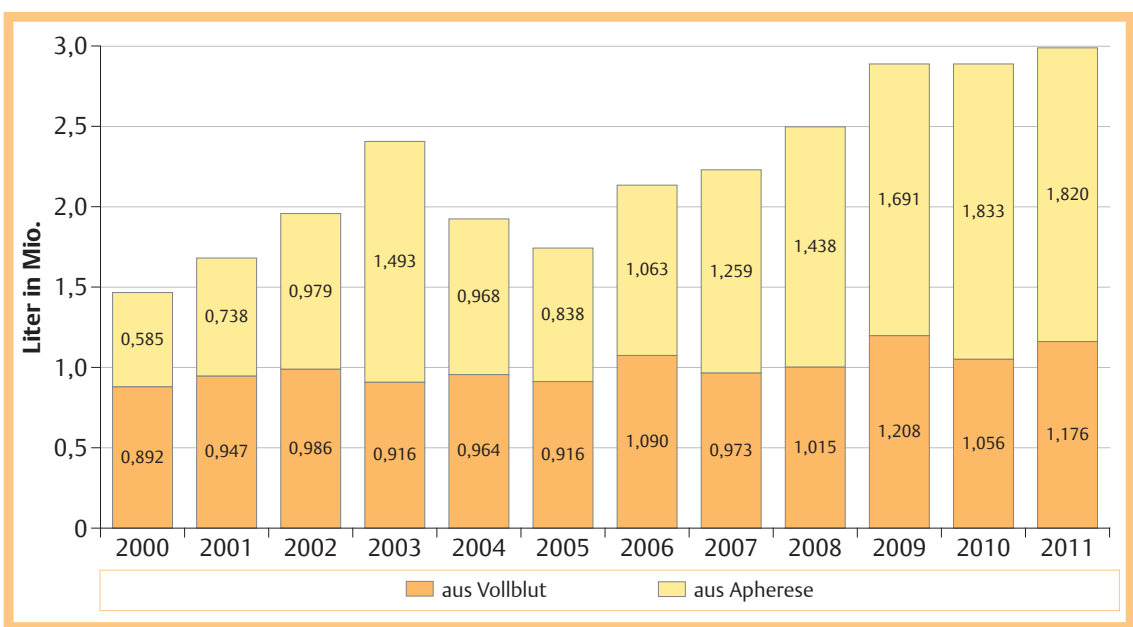

Abb. 5 Gewinnung von Plasma zur Fraktionierung (Quelle: PEI).

nach $\S 21$ Abs. 1 TFG die erforderlichen Angaben an das PEI, das die Daten anonymisiert in einem Bericht zusammenfasst und dann bekannt macht. Die 0 Abb. 4 u. 5 zeigen Übersichten, die verdeutlichen, dass die Gewinnung von Blut und Plasma nahezu kontinuierlich angestiegen ist und heute einen hohen Stand erreicht hat. Damit kann nicht nur der eigene Bedarf in Deutschland gedeckt werden, sondern es könnte auch anderen Mitgliedstaaten in der EU geholfen werden. Damit ist dem Gesichtspunkt der Selbstversorgung in der EU mit Blut und Plasma Rechnung getragen. Darüber hinaus ist ein hohes Spendenaufkommen auch wegen der demografischen Entwicklung und der Multimorbidität immer mehr älterer Menschen auch in Zukunft wichtig.

Das hohe Aufkommen an Blut und Plasma ist in erster Linie den spendenden Personen zu verdanken, die sich in den Dienst von Menschen stellen, die auf hochwertige Blutprodukte angewiesen sind. Diese Bereitschaft kann nicht hoch genug eingeschätzt und gewürdigt werden. Darüber hinaus bedarf es engagierter Spendeeinrichtungen, die sachkundig und auf hohem medizinischen und technischen Stand Blut und Plasma gewinnen und verarbeiten. Dasselbe gilt für die Plasma verarbeitende Industrie. In Deutschland hat sich auf der Grundlage eines modernen Arzneimittel- und Transfusionsrechts ein hochwertiges Blutspendewesen entwickelt. Es stellt nicht nur sichere Blutprodukte in ausreichendem Maße zur Verfügung, sondern beliefert auch eine hoch entwickelte Industrie mit Plasma zur Herstellung schwieriger, aber dringend benötigter Plasmaprodukte. Für das Spendenaufkommen sorgen die Spendeeinrichtungen u. a. mit verschiedenen auch öffentlich geförderten Formen der Spenderwerbung.
Die Bundesregierung unterstützt das Aufkommen an Blut und Plasma mit einer Motivationskampagne zur Blut- und Plasmaspende, die seit 1998 mit einer kurzfristigen Unterbrechung von der Bundeszentrale für gesundheitliche Aufklärung durchgeführt wird. Die Aufmachung der Internetseite macht deutlich, dass junge Spender angesprochen werden sollen. Die demografische Entwicklung führt dazu, dass der Anteil älterer Menschen bei der Blut- und Plasmaspende abnimmt. Das kann nur dadurch aufgefangen werden, dass der Anteil der jungen Spender erhöht wird.

\section{Zusammenfassung}

Das Transfusionsgesetz hat sich bewährt. Auf seiner Grundlage werden hochwertige und sichere Blutprodukte gewonnen und hergestellt. Mit dem Hämovigilanzsystem in TFG und AMG ist eine wesentliche Grundlage zur Vermeidung der Übertragung von Krankheiten durch Blutprodukte geschaffen worden. Das System zur Qualitätssicherung der Anwendung von Blutprodukten hat dazu beigetragen, dass heute die Behandlung der Patientinnen und Patienten in den Einrichtungen der Krankenversorgung auf höchst möglichem Niveau stattfindet. Das kann aufgrund der Ereignisse im Zusammenhang mit der Übertragung von HIV durch Blutprodukte nicht hoch genug bewertet werden. Wie die auf der Basis des TFG erhobenen Daten ausweisen, steht genügend Blut und Plasma zur Verfügung, um den Bedarf zur Versorgung der Bevölkerung mit Blutprodukten zu decken. Deutschlands Blutspende- und Transfusionswesen erfüllt höchste Ansprüche und ist Spitze in der Europäischen Union.

Literatur und Anmerkungen

1 Gesetz zur Regelung des Transfusionswesens (Transfusionsgesetz) in der Fassung der Bekanntmachung vom 28. August 2007 (BGBl. I S. 2169), geändert durch Artikel 12 des Gesetzes vom 17. Juli 2009 (BGBl. I S. 1990)

2 Bericht vom 21. Oktober 1994, BT-Drs. $12 / 8591$

3 Richtlinie 2002/98/EG des Europäischen Parlaments und des Rates vom 27. Januar 2003 zur Festlegung von Qualitäts- und Sicherheitsstandards für die Gewinnung, Testung, Verarbeitung, Lagerung und Verteilung von menschlichem Blut und Blutbestandteilen und zur Änderung der Richtlinie 2001/83/EG, ABl. EU Nr. L 33 S. 30

4 Richtlinie 2004/33/EG der Kommission vom 22. März 2004 zur Durchführung der Richt- 
linie 2002/98/EG des Europäischen Parlaments und des Rates hinsichtlich bestimmter technischer Anforderungen für Blut und Blutbestandteile, ABl. EU Nr. L 91 S. 25

5 Richtlinie 2005/61/EG der Kommission vom 30. September 2005 zur Durchführung der Richtlinie 2002/98/EG des Europäischen Parlaments und des Rates in Bezug auf die Anforderungen an die Rückverfolgbarkeit und die Meldung ernster Zwischenfälle und ernster unerwünschter Reaktionen, ABl. EU Nr. L 256 S. 32

6 Richtlinie 2005/62/EG der Kommission vom 30. September 2005 zur Durchführung der Richtlinie 2002/98/EG des Europäischen Parlaments und des Rates in Bezug auf gemeinschaftliche Standards und Spezifikationen für ein Qualitätssystem für Blutspendeeinrichtungen, ABl. EU Nr. L 256 S. 41

7 Gesetz über den Verkehr mit Arzneimitteln (Arzneimittelgesetz) in der Fassung der Be- kanntmachung vom 12. Dezember 2005 (BGBl. I S. 3394), zuletzt geändert durch Artikel 2 des Gesetzes vom 19. Oktober 2012 (BGBl. I S. 2192)

8 Arzneimittel- und Wirkstoffherstellungsverordnung vom 3. November 2006 (BGBL. I S. 2523), zuletzt geändert durch Artikel 10 des Gesetzes vom 19. Oktober 2012 (BGBl. I S. 2192)

9 Richtlinien zur Gewinnung von Blut und Blutbestandteilen und zur Anwendung von Blutprodukten (Hämotherapie) in der Fassung der Bekanntmachung vom 09.07.2010 (Bundesanzeiger Nr. 101a); vgl. hierzu auch von Auer, Zum Verständnis der Rechtsverordnungsermächtigung nach $\S 12$ Transfusionsgesetz (TFG) und der Richtlinienkompetenz nach $\S 12 \mathrm{a}$ TFG, in Transfus Med Hemother 2011; 38: 331-333

10 Querschnitts-Leitlinien (BÄK) zur Therapie mit Blutkomponenten und Plasmaderiva- ten, Gesamtnovelle 2008, zuletzt geändert am 01.08.2011

11 vgl. Merkblatt zur Qualitätssicherung in der Hämotherapie, Stand: Dezember 2012, der Ärztekammer Nordrhein; Musterarbeitsanweisung zur Transfusion von Erythrozytenkonzentraten, Stand: 2010, der Bundesärztekammer

12 Siehe Anmerkung 10

13 z.B. Checkliste Klinische Hämotherapie, Stand: 30.01.2007, des Berufsverbandes Deutscher Transfusionsmediziner (BDT); Qualitätsmanagementhandbuch, Stand: 01.05.2012, der Universitätsmedizin Greifswald

Friedger von Auer, Königswinter 